\title{
Nonlocal Variational Image Deblurring Models in the Presence of Gaussian or Impulse Noise
}

\author{
Miyoun Jung and Luminita A. Vese \\ University of California, Los Angeles, \\ Department of Mathematics, \\ Los Angeles, CA 90095-1555, USA \\ gomtaeng@math.ucla.edu, lvese@math.ucla.edu
}

\begin{abstract}
We wish to recover an image corrupted by blur and Gaussian or impulse noise, in a variational framework. We use two data-fidelity terms depending on the noise, and several local and nonlocal regularizers. Inspired by Buades-Coll-Morel, Gilboa-Osher, and other nonlocal models, we propose nonlocal versions of the Ambrosio-Tortorelli and Shah approximations to Mumford-Shah-like regularizing functionals, with applications to image deblurring in the presence of noise. In the case of impulse noise model, we propose a necessary preprocessing step for the computation of the weight function. Experimental results show that these nonlocal MS regularizers yield better results than the corresponding local ones (proposed for deblurring by Bar et al.) in both noise models; moreover, these perform better than the nonlocal total variation in the presence of impulse noise. Characterization of minimizers is also given.
\end{abstract}

\section{Introduction}

We consider the problem of restoring an image blurred and then contaminated by Gaussian or impulse noise. Let $f, u: \Omega \rightarrow \mathbb{R}$ be image intensity functions, where $\Omega \subset \mathbb{R}^{2}$ is open and bounded. The standard linear degradation model is $f=k * u+n ; f$ is the observed blurry-noisy image, $k$ is (known) spaceinvariant blurring kernel, $u$ is the ideal image we want to recover, and $n$ is additive random noise independent of $u$. We approach the restoration problem within the variational framework: $\inf _{u}\{\Phi(f-k * u)+\Psi(|\nabla u|)\}$, where $\Phi$ defines a data-fidelity term, and $\Psi$ defines the regularization that enforces a smoothness constraint on $u$, depending on its gradient $\nabla u$.

First, two different fidelity terms can be considered based on the noise; in the case of Gaussian noise model, the $L^{2}$-fidelity term led by the maximum likelihood estimation is commonly used: $\Phi(f-k * u)=\int_{\Omega}(f-k * u)^{2} d x$. However, the quadratic data fidelity term considers the impulse noise, which might be caused by bit errors in transmissions or wrong pixels, as an outlier. So, for the impulse noise model, the $L^{1}$-fidelity term is more appropriate, due to its robustness of removing outlier effects [2], [17]: $\Phi(f-k * u)=\int_{\Omega}|f-k * u| d x$.

Image deblurring-denoising is an inverse problem, which is known to be ill-posed due to either the non-uniqueness of the solution or the numerical 
instability of the inversion of the blurring operator. The regularization term $\Psi$ alleviates this problem by reflecting some a-priori properties. Several regularization terms were suggested in the literature, including [23, [9], 19], 20], 16]. Here, we consider the total variation regularization [19], [20] and two approximations of Mumford-Shah regularizers [16], denoted $M S H^{1}$ and $M S T V$, proposed by Ambrosio-Tortorelli [3] and Shah [21, [1] respectively and recently used for image deblurring in the presence of Gaussian and impulse noise by Bar et al [4, [5]. These traditional regularization terms are based on local image operators, which denoise and preserve edges very well, but may induce loss of fine structures like texture during the restoration process.

Recently, Buades et al [8] introduced the nonlocal means filter, which produces excellent denoising results. Kindermann et al [13] and Gilboa-Osher [10,11] formulated the variational framework of NL-means by proposing nonlocal regularizing functionals. Lou et al [14] used the nonlocal total variation $(N L / T V)$ of Gilboa-Osher in image deblurring in the presence of Gaussian noise with a preprocessing step for the computation of the weight function.

We propose here nonlocal versions of the approximated Mumford-Shah and Ambrosio-Tortorelli regularizing functionals, called $N L / M S H^{1}$ and $N L / M S T V$, by applying the nonlocal operators proposed by Gilboa-Osher to $M S H^{1}$ and $M S T V$ respectively, for image restoration in the presence of blur and Gaussian or impulse noise. In addition, for the impulse noise model, we propose to use a preprocessed image to compute the weights $w$ (the weights $w$ defined in the NL-means filter are more appropriate for the additive Gaussian noise). We note that the interesting parallel work [7] also proposed $N L / M S H^{1}$ regularizer for segmentation and denoising in the presence of Gaussian noise, but not for deblurring, nor for the impulse noise case. More details about our proposed methods are presented in [12].

Local Regularizers. In this section, we recall several regularization terms. The first one is the Mumford-Shah regularizing functional [16] which gives preference to piecewise smooth images. The MS regularizer, depending on the image $u$ and on its edge set $K \subset \Omega$, is given by $\Psi^{M S}(u, K)=\beta \int_{\Omega \backslash K}|\nabla u|^{2} d x+\alpha \int_{K} d \mathcal{H}^{1}$, where $\mathcal{H}^{1}$ is the 1D Hausdorff measure. The first term enforces smoothness of $u$ everywhere except on the edge set $K$, and the second one minimizes the total length of edges. But it is difficult to minimize in practice the non-convex MS functional. Ambrosio and Tortorelli [3] approximated this functional by a sequence of regular functionals $\Psi_{\epsilon}$ using the $\Gamma$-convergence. The edge set $K$ is represented by a smooth auxiliary function $v$. Thus we have an approximation to $\Psi^{M S}$ as 3

$$
\Psi_{\epsilon}^{M S H^{1}}(u, v)=\beta \int_{\Omega} v^{2}|\nabla u|^{2} d x+\alpha \int_{\Omega}\left(\epsilon|\nabla v|^{2}+\frac{(v-1)^{2}}{4 \epsilon}\right) d x,
$$

where $0 \leq v(x) \leq 1$ represents the edges: $v(x) \approx 0$ if $x \in K$ and $v(x) \approx 1$ otherwise, $\epsilon>0$ is a parameter, and $\alpha, \beta>0$. A minimizer $u=u_{\epsilon}$ of $\Psi_{\epsilon}^{M S H^{1}}$ approaches a minimizer $u$ of $\Psi^{M S}$ as $\epsilon \rightarrow 0$. 
An alternative approach is the total variation [19, 20, proposed by Rudin, Osher, and Fatemi, called $T V$ regularizer: $\Psi^{T V}(u)=\int_{\Omega}|D u| \approx \int_{\Omega}|\nabla u| d x$. Because of its benefits of preserving edges (which have high gradient levels) and convexity, $T V$ has been widely used in image restoration.

Shah 21] suggested a modified version of the AT approximation to the MS functional by replacing the 2-norm of $|\nabla u|$ by the 1-norm in the first term:

$$
\Psi_{\epsilon}^{M S T V}(u, v)=\beta \int_{\Omega} v^{2}|\nabla u| d x+\alpha \int_{\Omega}\left(\epsilon|\nabla v|^{2}+\frac{(v-1)^{2}}{4 \epsilon}\right) d x .
$$

This functional $\Gamma$-converges to the other functional $\Psi^{M S T V}$ as $\epsilon \rightarrow 0$, [1]:

$$
\Psi^{M S T V}(u)=\beta \int_{\Omega \backslash K}|\nabla u| d x+\alpha \int_{K} \frac{\left|u^{+}-u^{-}\right|}{1+\left|u^{+}-u^{-}\right|} d \mathcal{H}^{1}+\left|D_{c} u\right|(\Omega)
$$

where $u^{+}$and $u^{-}$denote the image values on two sides of the jump set $K=K_{u}$ of $u$, and $D_{c} u$ is the Cantor part of the measure-valued derivative $D u$. Note that the non-convex term $\frac{\left|u^{+}-u^{-}\right|}{1+\left|u^{+}-u^{-}\right|}$is similar with the prior regularization by Geman-Reynolds [9]. We observe that this regularizing functional is similar to the total variation of $u \in B V(\Omega)$ that can be written as $\int_{\Omega}|D u|=$ $\int_{\Omega \backslash K_{u}}|\nabla u| d x+\int_{K_{u}}\left|u^{+}-u^{-}\right| d \mathcal{H}^{1}+\left|D_{c} u\right|(\Omega)$. By comparing the second terms, we see that the MSTV regularizer does not penalize the jump part as much as the $T V$ regularizer. In this paper, we consider the $T V$ regularizer $\Psi^{T V}$, the $M S H^{1}$ regularizer $\Psi_{\epsilon}^{M S H^{1}}$, and the $M S T V$ regularizer $\Psi_{\epsilon}^{M S T V}$.

Nonlocal Regularizers. Nonlocal methods in image processing have been explored in many papers because they are well adapted to texture denoising while the standard denoising models working with local image information seem to consider texture as noise, which results in losing details. Nonlocal methods are generalized from neighborhood filters (e.g. Yaroslavsky filter, 24]) and patch based methods. The idea of neighborhood filter is to restore a pixel by averaging the values of neighboring pixels with a similar grey level value. Buades et al. 8 generalized this idea by applying the patch-based method, and proposed the famous nonlocal-means (or NL-means) filter for denoising, given by $N L u(x)=$ $\frac{1}{C(x)} \int_{\Omega} e^{-\frac{d_{a}(u(x), u(y))}{h^{2}}} u(y) d y ; d_{a}(u(x), u(y))=\int G_{a}(t)|u(x+t)-u(y+t)|^{2} d t$ is the patch distance, $G_{a}$ is the Gaussian kernel with standard deviation $a$ determining the patch size, $C(x)=\int_{\Omega} e^{-\frac{d_{a}(u(x), u(y))}{h^{2}}} d y$ is a normalization factor, and $h$ is the filtering parameter corresponding to the noise level (usually the standard deviation of the noise). The NL-means not only compares the grey level at a single point but the geometrical configuration in a whole neighborhood (patch).

In the variational framework, Kindermann et al 13 formulated the neighborhood filters and NL-means filters as nonlocal regularizing functionals which generally are not convex. Then, Gilboa-Osher [10] formalized the convex nonlocal functional inspired from graph theory, and moreover, based on the gradient and divergence definitions on graphs in the context of machine learning, 
they [11 derived the corresponding nonlocal operators. Let $u: \Omega \rightarrow \mathbb{R}$ be a function, and $w: \Omega \times \Omega \rightarrow \mathbb{R}$ be a nonnegative and symmetric weight function. The nonlocal gradient vector $\nabla_{w} u: \Omega \times \Omega \rightarrow \mathbb{R}$ is $\left(\nabla_{w} u\right)(x, y):=$ $(u(y)-u(x)) \sqrt{w(x, y)}$. Hence, the nonlocal divergence $\operatorname{div}_{w} \vec{v}: \Omega \rightarrow \mathbb{R}$ of the vector $\vec{v}: \Omega \times \Omega \rightarrow \mathbb{R}$ is defined as the adjoint of the nonlocal gradient, $\left(\operatorname{div}_{w} \vec{v}\right)(x):=\int_{\Omega}(v(x, y)-v(y, x)) \sqrt{w(x, y)} d y$, and the norm of the nonlocal gradient of $u$ at $x \in \Omega$ is given by $\left|\nabla_{w} u\right|(x)=\sqrt{\int_{\Omega}(u(y)-u(x))^{2} w(x, y) d y}$. Based on these nonlocal operators, they introduced nonlocal regularizing functionals of the general form $\Psi(u)=\int_{\Omega} \phi\left(\left|\nabla_{w} u\right|^{2}\right) d x$, where $s \mapsto \phi(s)$ is a positive function, convex in $\sqrt{s}$, and $\phi(0) \stackrel{=}{=}$. By taking $\phi(s)=\sqrt{s}$, they proposed the nonlocal TV regularizer $(N L / T V)$ which corresponds in the local case to $\Psi^{T V}(u)=\int_{\Omega}|\nabla u| d x$. Inspired by these ideas, we propose in the next section nonlocal versions of Ambrosio-Tortorelli and Shah approximations to the MS regularizers for image denoising-deblurring. This is also continuation of the work by Bar et al. 4], 5], first to propose the use of Mumford-Shah-like approximations to image restoration.

In practice, we use the search window $\Omega_{w}=\{y \in \Omega:|y-x| \leq r\}$ instead of $\Omega$ (semi-local) and the weight function $w$ at $(x, y) \in \Omega \times \Omega$ depending on a function $f: \Omega \rightarrow \mathbb{R}, w(x, y)=\exp \left(-\frac{d_{a}(f(x), f(y))}{h^{2}}\right)$. The weight function $w(x, y)$ gives the similarity of image features between two pixels $x$ and $y$, which is normally computed using the blurry-noisy image $f$. Recently, for image deblurring in the presence of Gaussian noise, Lou et al [14 used a preprocessed image obtained by applying the Wiener filter to $f$, instead of $f$, to compute $w$. In our work, only for the impulse noise model, we propose a different preprocessing step and evaluate $w$ by using the preprocessed image.

\section{Description of the Proposed Models}

We propose the following nonlocal Mumford-Shah regularizers $(N L / M S)$ by applying the nonlocal operators to the approximations of the MS regularizer

$$
\Psi^{N L / M S}(u, v)=\beta \int_{\Omega} v^{2} \phi\left(\left|\nabla_{w} u\right|^{2}\right) d x+\alpha \int_{\Omega}\left(\epsilon|\nabla v|^{2}+\frac{(v-1)^{2}}{4 \epsilon}\right) d x,
$$

where $\phi(s)=s$ and $\phi(s)=\sqrt{s}$ correspond to the nonlocal versions of $M S H^{1}$ and $M S T V$ regularizers, so called $N L / M S H^{1}$ and $N L / M S T V$, respectively. In addition, we use these nonlocal regularizers to deblur images in the presence of Gaussian or impulse noise. Thus, by incorporating the proper fidelity term depending on the noise model, we design two types of total energies as

$$
\begin{aligned}
& \text { Gaussian noise model: } E^{G}(u, v)=\int_{\Omega}(f-k * u)^{2} d x+\Psi^{N L / M S}(u, v), \\
& \text { Impulse noise model: } E^{I m}(u, v)=\int_{\Omega}|f-k * u| d x+\Psi^{N L / M S}(u, v) .
\end{aligned}
$$


Minimizing these functionals in $u$ and $v$, we obtain the Euler-Lagrange equations

$$
\frac{\partial E^{G}}{\partial v}=\frac{\partial E^{I m}}{\partial v}=2 \beta v \phi\left(\left|\nabla_{w} u\right|^{2}\right)-2 \epsilon \alpha \Delta v+\alpha\left(\frac{v-1}{2 \epsilon}\right)=0,
$$

Gaussian noise model: $\quad \frac{\partial E^{G}}{\partial u}=\tilde{k} *(k * u-f)+L^{N L / M S} u=0$,

$$
\text { Impulse noise model: } \quad \frac{\partial E^{I m}}{\partial u}=\tilde{k} * \operatorname{sign}(k * u-f)+L^{N L / M S} u=0,
$$

where $\tilde{k}(x)=k(-x)$ and

$$
\begin{aligned}
L^{N L / M S} u= & -2 \int_{\Omega}\{(u(y)-u(x)) w(x, y) \\
& \cdot\left[\left(v^{2}(y) \phi^{\prime}\left(\left|\nabla_{w}(u)\right|^{2}(y)\right)+v^{2}(x) \phi^{\prime}\left(\left|\nabla_{w}(u)\right|^{2}(x)\right)\right]\right\} d y .
\end{aligned}
$$

The energy functionals $E^{G}(u, v)$ and $E^{I m}(u, v)$ are convex in each variable and bounded from below. Therefore, to solve two Euler-Lagrange equations simultaneously, the alternate minimization approach is applied. Note that since both energy functionals are not convex in the joint variable $(u, v)$, we may compute only a local minimizer. However, this is not a drawback in practice, since the initial guess for $u$ in our algorithm is the data $f$.

To extend the nonlocal methods to the impulse noise case, we need a preprocessing step for the weight function $w$ since we cannot directly use the data $f$ to compute $w$. In other words, in the presence of impulse noise, the noisy pixels tend to have larger weights than the other neighboring points, so it is likely to keep the noise value at such pixel. Thus, we propose a simple algorithm to obtain a preprocessed image $g$, which removes the impulse noise (outliers) as well as preserving texture as much as possible. Basically, we use the median filter, well-known for removing impulse noise. However, if we apply one-step of the median filter, then the output may be too smoothed out. In order to preserve fine structures as well as to remove noise properly, we take the idea of Bregman iteration [6], [18, and we propose the following algorithm to obtain a preprocessed image $g$ that will be used only in the computation of the weight function $w$ :

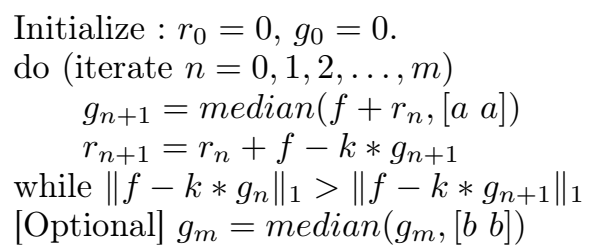

where $f$ is the given noisy-blurry data, median $(f,[a \quad a])$ is the median filter of size $a \times a$ with input $f$; the optional step is needed in the case when the final $g_{m}$ still has some salt-and-pepper-like noise. This algorithm is simple, it requires a few iterations only, and it takes less than 1 second for a $256 \times 256$ size image. Moreover, the preprocessed image $g_{m}$ is a deblurred and denoised version of $f$; it will be used only in the computation of the weights $w$, while keeping $f$ in the data fidelity term, thus artifacts are not introduced by the median filter. 
Characterization of Minimizers. In this section we characterize the minimizers of the functionals formulated with the nonlocal regularizers, using [15, 22]. Assuming that a functional $\|\cdot\|$ on a subspace of $L^{2}(\Omega)$ is a semi-norm, we can define the dual norm (where $\langle\cdot, \cdot\rangle$ denotes the $L^{2}(\Omega)$ inner product) of $f \in L^{2}(\Omega) \subset L^{1}(\Omega)$ as $\|f\|_{*}:=\sup _{\|\varphi\| \neq 0} \frac{\langle f, \varphi\rangle}{\|\varphi\|} \leq+\infty$, so that the usual duality $\langle f, \varphi\rangle \leq\|\varphi\|\|f\|_{*}$ holds for $\|\varphi\| \neq 0$. We define two functionals (here $K u:=k * u)$,

$$
\begin{aligned}
F(u) & =\lambda \int_{\Omega}|f-K u|^{2} d x+|u|_{N L / T V}, \\
G(u, v) & =\int_{\Omega} \sqrt{|f-K u|^{2}+\eta^{2}} d x+\beta|u|_{N L / M S}+\alpha\left(\int_{\Omega}\left(\epsilon|\nabla v|^{2}+\frac{|v-1|^{2}}{4 \epsilon}\right) d x\right)
\end{aligned}
$$

where $\lambda>0$, and $|u|_{N L / M S} \in\left\{|u|_{N L / M S T V, v},|u|_{N L / M S H^{1}, v}\right\}$. We use here the notations $|u|_{N L T V}=\int_{\Omega}\left|\nabla_{w} u\right|(x) d x,|u|_{N L / M S T V, v}=\int_{\Omega} v^{2}(x)\left|\nabla_{w} u\right|(x) d x$, and $|u|_{N L / M S H^{1}, v}=\sqrt{\int_{\Omega} v^{2}(x)\left|\nabla_{w} u\right|^{2}(x) d x}$, which are semi-norms. We modified the regularizing functional $|u|_{N L / M S H^{1}, v}$; the square-root term replaces the original term of our model, $\int_{\Omega} v^{2}(x)\left|\nabla_{w} u\right|^{2}(x) d x$. It is introduced here to enable the characterization of minimizers below, but the numerical calculations utilize the original formulation. For the proofs we refer to [12.

Proposition 1. Let $K: L^{2}(\Omega) \rightarrow L^{2}(\Omega)$ be a linear bounded blurring operator with adjoint $K^{*}$ and let $F$ be the associated functional. Then

(1) $\left\|K^{*} f\right\|_{*} \leq \frac{1}{2 \lambda}$ if and only if $u \equiv 0$ is a minimizer of $F$.

(2) Assume that $\frac{1}{2 \lambda}<\left\|K^{*} f\right\|_{*}<\infty$. Then $u$ is a minimizer of $F$ if and only if $\left\|K^{*}(f-K u)\right\|_{*}=\frac{1}{2 \lambda}$ and $\left\langle u, K^{*}(f-K u)\right\rangle=\frac{1}{2 \lambda}|u|_{N L / T V}$,

where $\|\cdot\|_{*}$ is the corresponding dual norm of $|\cdot|_{N L / T V}$.

Proposition 2. Let $K: L^{2}(\Omega) \rightarrow L^{2}(\Omega)$ be a linear bounded blurring operator with adjoint $K^{*}$ and let $G$ be the associated functional. If $(u, v)$ is a minimizer of $G$ with $v \in[0,1]$, then

$\left\|K^{*} \frac{f-K u}{\sqrt{(f-K u)^{2}+\eta^{2}}}\right\|_{*}=\beta$ and $\left\langle K^{*} \frac{f-K u}{\sqrt{(f-K u)^{2}+\eta^{2}}}, u\right\rangle=\beta|u|_{N L / M S}$,

where $\|\cdot\|_{*}$ is the corresponding dual norm of $|\cdot|_{N L / M S}$.

\section{Experimental Results and Comparisons}

The nonlocal MS regularizers proposed here, $N L / M S T V$ and $N L / M S H^{1}$, are tested on several images with different blur kernels and noise types. We compare them with their traditional (local) versions, such as $M S T V$ and $M S H^{1}$, and with the local and nonlocal total variations ( $T V$ [20, $N L / T V$ [1]). In addition, we experiment the nonlocal regularizers in the impulse noise model with a preprocessing step for the weight function. 


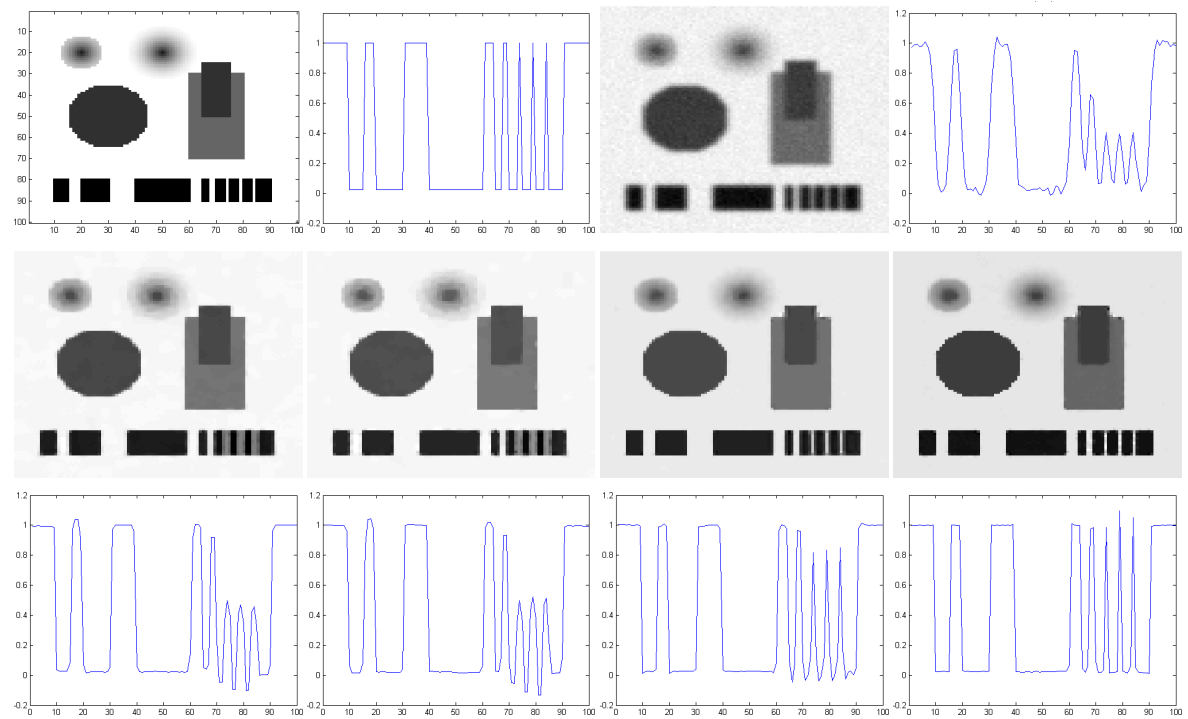

Fig. 1. Image recovery with cross sections: Gaussian blur kernel with $\sigma_{b}=1$ and Gaussian noise with $\sigma_{n}=5$. Top: original image and its cross section, noisy blurry image and its cross section. Middle, Bottom rows: recovered images (middle) and recovered cross sections (bottom) using $T V, M S T V, N L / T V, N L / M S T V$. SNR for the results: $T V=32.9485, M S T V=33.5629, N L / T V=45.1943, N L / M S T V=50.6618$. $\beta=0.0045(M S T V), 0.001(N L / M S T V), \alpha=0.00000015, \epsilon=0.000001$.

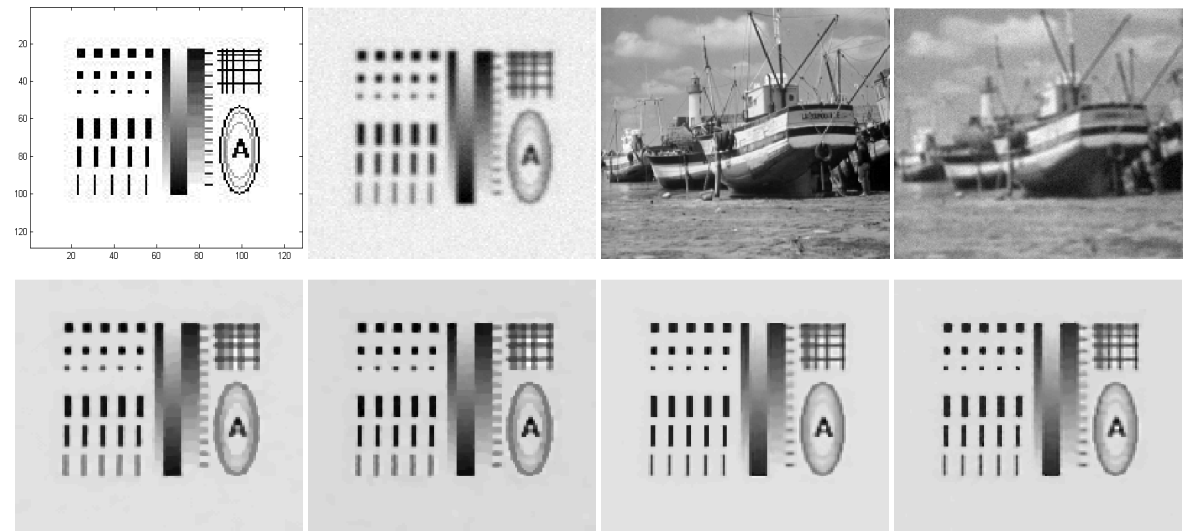

Fig. 2. Top: (1st, 3rd) original images, (2nd, 4th) noisy blurry images with Gaussian kernel with $\sigma_{b}=1$ (2nd) and using the pill-box kernel of radius 2 (4th), and then contaminated by Gaussian noise with $\sigma_{n}=5$. Bottom: recovered images with SNR values: $T V$ (14.4240), MSTV (14.4693), NL/TV (17.4165), NL/MSTV (16.5776). $\beta=0.007, \alpha=0.00000015(M S T V), \beta=0.0025, \alpha=0.00000025$ (NL/MSTV), $\epsilon=0.0000005$. 


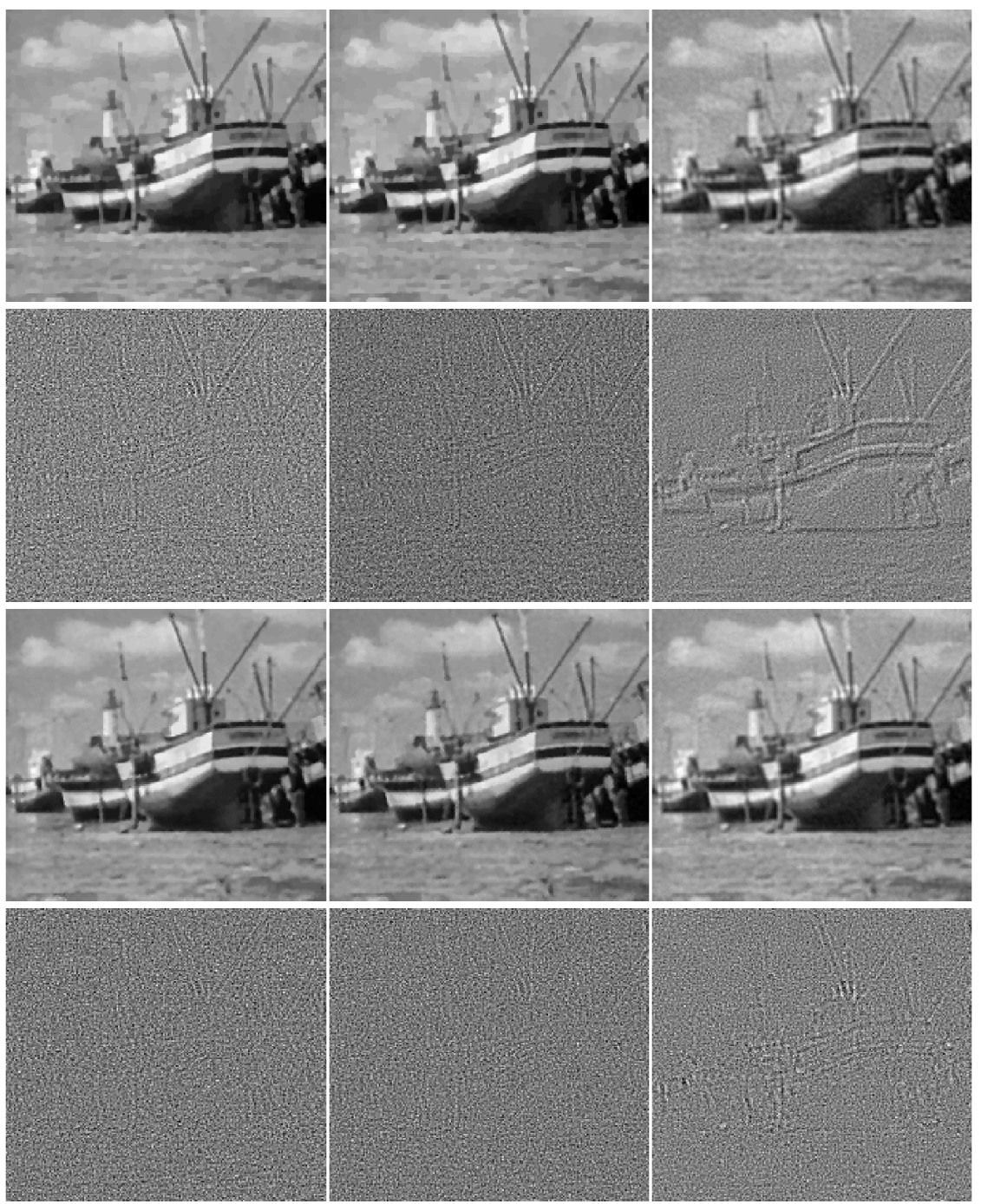

Fig. 3. Recovery of noisy blurry image from Fig. 3. Top: recovered image $u$ using $T V$ $(\mathrm{SNR}=25.0230), M S T V \quad(\mathrm{SNR}=25.1968), M S H^{1} \quad(\mathrm{SNR}=23.1324)$. Third row: recovered image $u$ using $N L / T V(\mathrm{SNR}=26.4554), N L / M S T V(\mathrm{SNR}=26.4696), N L / M S H^{1}$ $(\mathrm{SNR}=24.7164)$. Second, bottom rows: corresponding residuals $f-k * u . \beta=0.0045$ $(M S T V), 0.001(N L / M S T V), 0.06\left(M S H^{1}\right), 0.006\left(N L / M S H^{1}\right), \alpha=0.00000001$, $\epsilon=0.00002$.

First, we test the Gaussian noise model in Figs. 1]3. As expected, $N L / M S T V$ and $N L / M S H^{1}$ perform better than $M S T V$ and $M S H^{1}$ respectively in the sense that not only they recover the fine scales such as texture better, but also in the case of $N L / M S T V$, the model does not produce any staircase effect (appeared in $M S T V$ ). Furthermore, comparing the nonlocal MS regularizers 

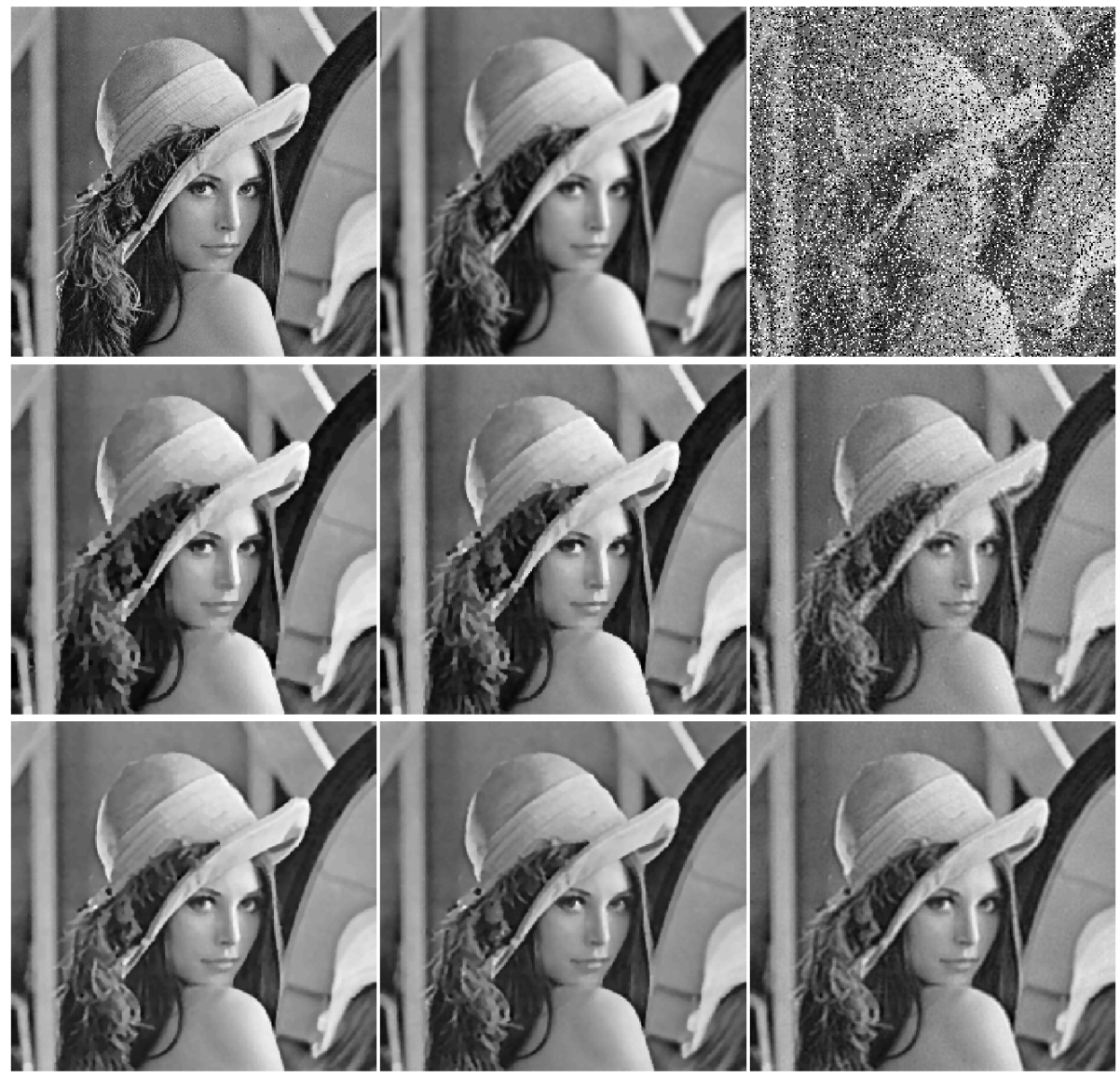

Fig. 4. Recovery of noisy blurry image with Gaussian kernel with $\sigma=1$ and saltand-pepper noise with $d=0.3$. Top: original image, blurry image, noisy-blurry image. Middle: recovered images using $T V(\mathrm{SNR}=26.9251), M S T V \quad(\mathrm{SNR}=27.8336)$, $M S H^{1} \quad(\mathrm{SNR}=23.2052)$. Bottom: recovered images using $N L / T V \quad(\mathrm{SNR}=29.2403)$, $N L / M S T V \quad(\mathrm{SNR}=29.3503), N L / M S H^{1} \quad(\mathrm{SNR}=27.1477)$. Second column: $\beta=0.25$ (MSTV), $0.1(N L / M S T V), \alpha=0.01, \epsilon=0.002$. Third column: $\beta=2\left(M S H^{1}\right), 0.55$ $\left(N L / M S H^{1}\right), \alpha=0.001, \epsilon=0.0001$.

with $N L / T V, N L / M S T V$ and $N L / T V$ seem to lead to similar results visually and according to SNR, while $N L / M S H^{1}$ gives a smoother image and lower SNR. Specifically, in Fig. 1, we use a simple image and its $1 D$ cross section. In this example, we use $11 \times 11$ size search window for $N L / M S T V$ which is sufficient to obtain the best result, while $N L / T V$ needs a $31 \times 31$ size. Moreover, $N L / M S T V$ recovers the signals much better than $N L / T V$, which might be caused by the fact that originally, $M S T V$ regularizer does not suppress the jump part as much as $T V$. On the other hand, in Fig. 2, $N L / T V$ produces clearer edges leading to higher SNR, while $N L / M S T V$ has some artifacts near the edges of especially 

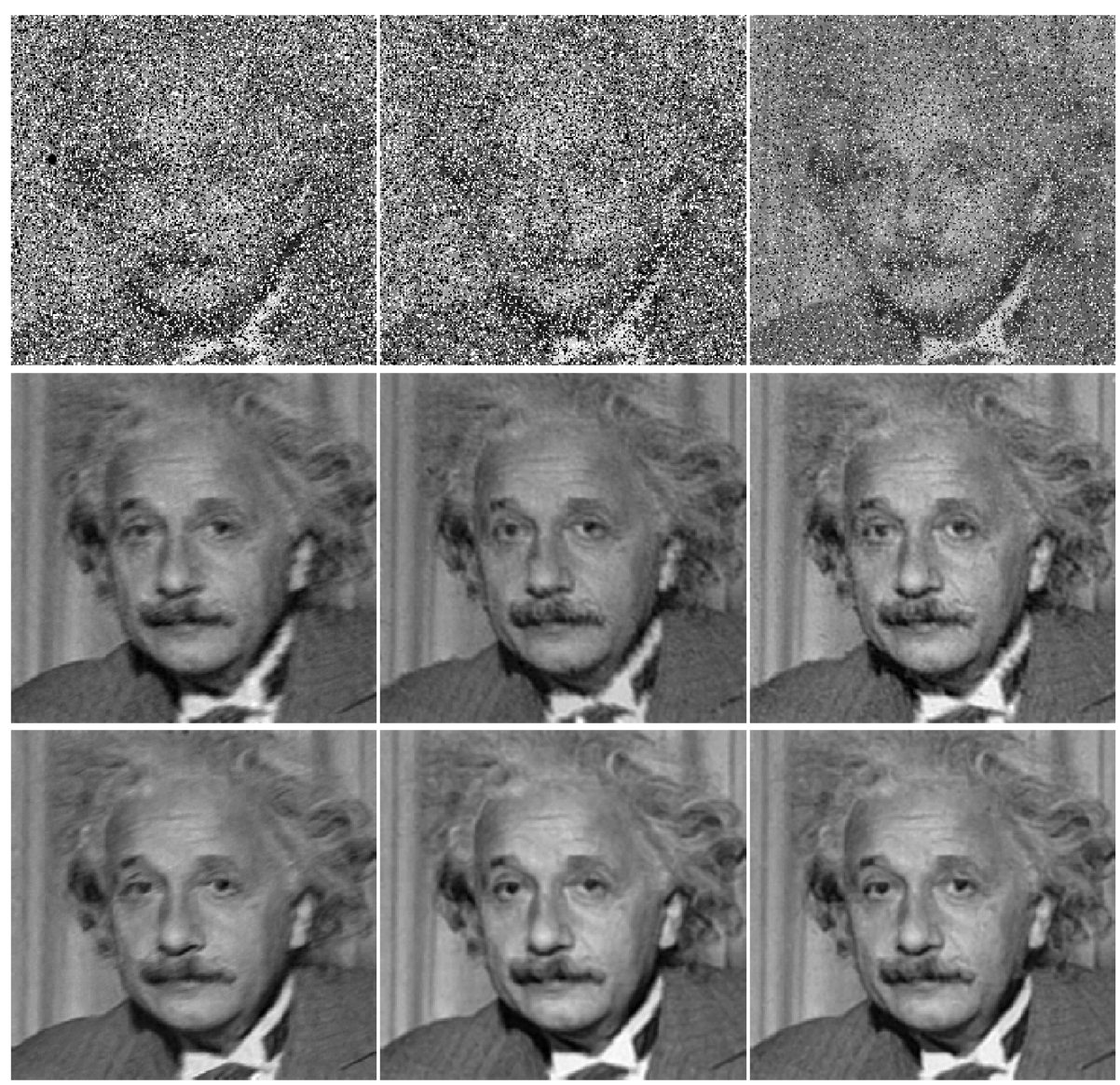

Fig. 5. Comparison between $M S H^{1}$ and $N L / M S H^{1}$ with the image blurred and contaminated by high density $(d=0.4)$ of impulse noise. Top: noisy blurry images (left) using motion blur kernel of length $=10$, oriented at angle $\theta=25^{\circ}$ w.r.t. the horizon and salt-and-pepper noise with $d=0.4$, (middle) using Gaussian kernel with $\sigma_{b}=1$ and salt-and-pepper noise with $d=0.4$, (right) using Gaussian kernel with $\sigma_{b}=1$ and random-valued impulse noise with $d=0.4$. Middle: recovered images using $M S H^{1}$, (left) $\mathrm{SNR}=17.1106$, (middle) $\mathrm{SNR}=15.2017$, (right) $\mathrm{SNR}=16.6960$. Bottom: recovered images using $N L / M S H^{1}$, (left) $\mathrm{SNR}=21.2464$, (middle) $\mathrm{SNR}=23.1998$, (right) $\mathrm{SNR}=24.2500$. First column: $\beta=2\left(M S H^{1}\right), 0.4\left(N L / M S H^{1}\right)$, second column: $\beta=2$ $\left(M S H^{1}\right), 1\left(N L / M S H^{1}\right), \alpha=0.001, \epsilon=0.0002$. Third column: $\beta=2.5\left(M S H^{1}\right)$, $0.65\left(N L / M S H^{1}\right), \alpha=0.000001, \epsilon=0.002$.

small black boxes. However, in the other real boat image, there is no significant difference between them visually and according to SNR (see Fig. 31). Fig. 3 also justifies the result that the nonlocal regularizers preserve edges and details better than the traditional local ones because we see less textures in the residuals $f-k * u$. 
Next, we recover a blurred image contaminated by impulse noise (salt-andpepper noise or random-valued impulse noise). First, we test all the nonlocal regularizers and the corresponding local ones on the Lenna image Fig. 4 with Gaussian blur kernel and salt-and-pepper noise with the noise density $d=0.3$, and then we test $M S H^{1}$ and $N L / M S H^{1}$ on the Einstein image Fig. 5 with different blur kernels and both impulse noise models, salt-and-pepper noise and random-valued impulse noise, with the same noise density $d=0.4$. By using a preprocessed image for the weight function, all the nonlocal regularizers outperform the traditional local ones by reducing the staircase effect and recovering the details better. Comparing the nonlocal regularizers, both $N L / T V$ and $N L / M S T V$ seem to give better results than $N L / M S H^{1}$ in the sense of SNR, but visually $N L / M S H^{1}$ looks more natural by preserving texture or details better especially with high noise density (see Fig. (4). Moreover, in the presence of high density of noise, $M S H^{1}$ suffers from restoring images especially blurred with Gaussian kernel, while it works satisfactorily with the other blur kernels such as motion blur. But, $N L / M S H^{1}$ performs very well with Gaussian blur as well as it produces better results with the other blur kernels. This can be seen in Figures 4 and 5. In Fig. 4 with Gaussian blur and high noise density $d=0.3$, $M S H^{1}$ suffers from some artifacts induced by noise, while $M S T V$ and $T V$ give cleaner results. On the other hand, $N L / M S H^{1}$ provides visually better result than the other nonlocal ones by preserving the fine structures. Even though $N L / M S T V$ gives the highest SNR, the result still looks more like cartoon by suppressing the texture parts especially in the hat part. So in this case, we visually prefer $N L / M S H^{1}$. Based on the above results, in Fig. [5, we only compare $M S H^{1}$ and $N L / M S H^{1}$ with the different blur kernels and both impulse noise models with higher noise density $d=0.4$. As expected, $N L / M S H^{1}$ produces better results than $M S H^{1}$ in both blur cases; especially in the Gaussian blur case, the results do not have any artifacts, unlike $M S H^{1}$.

Finally we note that in the MS regularizers, the parameters $\alpha, \beta$ and $\epsilon$ were selected manually to provide the best SNR results. The smoothness parameter $\beta$ increases with noise level while the other parameters $\alpha, \epsilon$ are approximately fixed. For the computational time, it takes about 5 minutes for constructing the weight function of a $256 \times 256$ image with the $11 \times 11$ search window and $5 \times 5$ patch in MATLAB on a dual core laptop with $2 \mathrm{GHz}$ processor and $2 \mathrm{~GB}$ memory. The minimization for the (local or nonlocal) MS regularizers takes around 60 seconds for the computations of both $u$ using an explicit scheme based on the gradient descent method and $v$ using a semi-implicit scheme with the total iterations $5 \times(100+5)$, while the (local or nonlocal) TV regularizer using gradient descent with an explicit scheme takes less than 55 seconds with 500 iterations.

\section{Acknowledgments}

This work has been supported by the National Science Foundation Grants DMS0714945 and DMS-0312222. 


\section{References}

1. Alicandro, R., Braides, A., Shah, J.: Free-discontinuity problems via functionals involving the $L^{1}$-norm of the gradient and their approximation. Interfaces Free Bound 1, 17-37 (1999)

2. Alliney, S.: Digital Filters as Absolute Norm Regularizers. IEEE TSP 40(6), 1548$1562(1992)$

3. Ambrosio, L., Tortorelli, V.M.: On the approximation of free discontinuity problems. Boll. Un. Mat. Ital. 6-B, 105-123 (1992)

4. Bar, L., Sochen, N., Kiryati, N.: Semi-Blind Image Restoration via Mumford-Shah Regularization. IEEE TIP 15(2), 483-493 (2006)

5. Bar, L., Sochen, N., Kiryati, N.: Image deblurring in the presence of impulsive noise. IJCV 70, 279-298 (2006)

6. Bregman, L.M.: The relaxation method for finding common points of convex sets and its application to the solution of problems in convex programming. USSR Computational Mathematics and Mathematical Physics 7, 200-217 (1967)

7. Bresson, X., Chan, T.F.: Non-local unsupervised variational image segmentation models. UCLA C.A.M. Report 08-67 (2008)

8. Buades, A., Coll, B., Morel, J.M.: A review of image denoising algorithms, with a new one. SIAM MMS 4(2), 490-530 (2005)

9. Geman, D., Reynolds, G.: Constrained Restoration and the Recovery of Discontinuities. IEEE TPAMI 14(3), 367-383 (1992)

10. Gilboa, G., Osher, S.: Nonlocal linear image regularization and supervised segmentation. SIAM MMS 6(2), 595-630 (2007)

11. Gilboa, G., Osher, S.: Nonlocal operators with applications to image processing. SIAM MMS 7(3), 1005-1028 (2008)

12. Jung, M., Vese, L.A.: Image restoration via nonlocal Mumford-Shah regularizers. UCLA C.A.M. Report 09-09 (2009)

13. Kindermann, S., Osher, S., Jones, P.W.: Deblurring and denoising of images by nonlocal functionals. SIAM MMS 4(4), 1091-1115 (2005)

14. Lou, Y., Zhang, X., Osher, S., Bertozzi, A.: Image recovery via nonlocal operators. UCLA C.A.M. Report 08-35 (2008)

15. Meyer, Y.: Oscillating Patterns in Image Processing and Nonlinear Evolution Equations. Univ. Lecture Ser. 22 (2002)

16. Mumford, D., Shah, J.: Optimal approximations by piecewise smooth functions and associated variational problems. Comm. Pure Appl. Math. 42, 577-685 (1989)

17. Nikolova, M.: Minimizers of cost-functions involving non-smooth data-fidelity terms. Application to the processing of outliers. SIAM Num. Anal. 40(3), 965-994 (2002)

18. Osher, S., Burger, M., Goldfarb, D., Xu, J., Yin, W.: An iterative regularization method for total variation based image restoration. SIAM MMS 4, 460-489 (2005)

19. Rudin, L., Osher, S., Fatemi, E.: Nonlinear total variation based noise removal algorithms. Phys. D 60, 227-238 (1996)

20. Rudin, L., Osher, S.: Total variation based image restoration with free local constraints. IEEE ICIP 1, 31-35 (1994)

21. Shah, J.: A common framework for curve evolution, segmentation and anisotropic diffusion. In: IEEE CVPR, pp. 136-142 (1996)

22. Tadmor, E., Nezzar, S., Vese, L.: Multiscale hierarchical decomposition of images with applications to deblurring. Denoising and segmentation: CMS 6(2), 281-307 (2008)

23. Tichonov, A., Arsensin, V.: Solution of ill-posed problems. Wiley, New York (1977)

24. Yaroslavsky, L.P.: Digital image processing: An Introduction. Springer, Heidelberg (1985) 